\title{
Beitrag zur Kenntnis der Schwanzbildung beim Menschen
}

\author{
Fuminori YANAGISAWA**
}

有尾人間（geschwänzter Mensch）の知見補遺

\begin{abstract}
柳沢 文 憲*
インドネシア南カリマンタンのバリト河上流のダナンパンガン地方のB村（本地方はイスラム信徒）飞住む 3 $1 / 3$ 才の有尾男児 (名N) の観察を括こなつた. その男児の父は 45 才, 農業を営み母は 35 才で第 1 子. 本児出生の

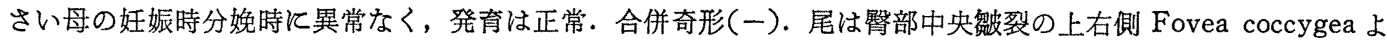
り発し, 脊椎との連係なく, 長さ $12 \mathrm{~cm}$, 基部の直径 $1 \mathrm{~cm}$, 最大径 $2 \mathrm{~cm}$, 随意運動はなく, 触診では骨, 筋肉を認 めず脂肪が主体である。外科的な切断は容易であるので申出でた所, 手術が全く不可能な精神世界を形成されて いた，尾は神からの授かりあので，本児は神童だからである(迷信とみなされよう).

人尾の定義は Bartels (1884), 分類はまた Bartels の5 型が慣用されている. 有尾人間の文献を現在まで狩 猟し紹介した。また古来カリマンタン（旧ボルネオ）の有尾人間についてはオランダ人により全世界でもっとも 多く報告されたところで，その真実性に対して賛否両論があるが，著者が現地で聞いた印象では, 現地人は有尾 人間を珍らしくないと見なしている.

〔本要旨は第36回日本民族衛生学会総会（昭46）飞発表した〕
\end{abstract}

\section{Einleitung}

Das Problem der geschwänzten Menschen hat von jeher sowohl die Anthropologen und Ärzte, als auch die Laien beschäftigt. Auf der einen Seite waren die Berichte über das Vorkommen des Schwanzmenschen insofern stark übertrieben, auf der anderen Seite wurde der geschwänzte Mensch vollständig als ein Fabel verwiesen. Die zahlreichen Mitteilungen stammen daher nur zum kleinen Teil von Ärzte und Naturforschern, zum grössten Teile von Reisenden, die in fremden Erdteilen Volksstämme aufgesucht und beschrieben haben. Max Bartels schreibt ein ausgezeichnetes Referat im Jahre 1884 und gibt die recht zutreffende Definition der Menschensçhwänze, die klare Schwanzeinteilung in 5 Formen und die geogra- phische Verbreitung der geschwänzten Menschen an. Er gibt wohl zum ersten Mal eine klare Definition, indem er unter einem Schwanz beim Menschen ein Gebilde versteht, “welches schon bei der Geburt des Menschen existierend, die hintere Längsachse des Körpers. über das hintere Körperende hinaus verlängert, so dass es scheinbar oder in Wirklichkeit eine Fortsetzung der Wirbelsäule über den Anfang der Hinterbacken hinaus nach abwärts bildet". Die von ihm gesammelter Fälle teilt er in 5 Formen ein. Jorns (1955) erklärt die Bartels' Einteilung klar. Die Beschreibung der Bartels* Einteilung ist nach Jorns folgende:

Erste Form. Echte Tierschwänze, gekennzeichnet durch deutlich differenziert und der Zahl nach vermehrte Wirbelkörper. Sowohl beim

* 東京医科歯科大学医学部農村厚生医学研究施設（主任：柳沢文徳教授）

**The Medical Institute for Rural Welfare, Tokyo Medical and Dental University 
Menschen wie beim Menschenaffen erfolgt eine Rückbildung der Schwanzwirbelsäule. Die Annahme eines phylogenetischen Rückschlages liegt daher nahe.

Zweite Form. Stummelschwänze ohne knöchernes Gerüst. Diese Schwänze stellen nach Form und Bau eine Wiederholung des Schwanzstummels dar, den der menschliche Embryo normalerweise in der Zeit nach der 3. Woche bis zu Beginn des 4. Monats seiner Entwicklung aufweist. Demnach eine Hemmungsmissbildung aus derjenigen Periode des intrauterinen Lebens, in welchem der Embryo geschwänzt ist.

Dritte Form. Angewachsene Schwänze, gekennzeichnet durch ihre dreiseitige Gestalt sowie durch ihre Lage in der Kreuz-Steissbeinregion, von der sie infolge Verwachsung mit den unterliegenden Teilen nicht abgehoben werden können. Bartels steht sie als Hemmungsbildung aus der Steiss-Höckerperiode der intrauterinen Entwicklung an.

Vierte Form. Lange dunne Schwänze, häufig von Schweineschwanzform (Abb. 1 u. 2,), gekennzeichnet durch ihre verhältnismässig grosse Länge, ihre selten gekrümmte Spitze und den völligen Mangel an differenzierten und verknöcherten Wirbeln. Ihr Auftreten müssen wir uns verursacht denken durch einen Prozess der Wachstumssteigerung, welche auf den Schwanz des Embryo einwirkte, so dass derselbe, anstatt zu schrumpfen und zu schwinden, im Gegenteil sich vergrösserte und ausdehnte und zu einem persistierenden Organe wurde. Die Zeit, in welche wir den die Wachsumtssteigerung bedingenden Reiz zu verlegen haben, kann selbstverständlich nur diejenige sein, in welcher der embryonale Schwanz normaler Weise existiert, das heisst nach der dritten Woche und vor Beginn des vierten Monats unserer intrauterinen Entwicklung.
Fünfte Form. Stummelschwänze mit deutlich differenzierten und verknöcherten, jedoch zahlenmässig nicht vermehrten Steissbeinwirbeln, deren Grösse aber, wohl infolge abnormen embryonalen Wachstums, gesteigert ist.

\section{Fallbericht}

Im Sommer 1971 war ich in Süd-Kalimantan/ Indonesien zwecks der medizinischen Untersuchungen im Kindesalter durch die Empfehlung der japanischen tropischen medizinischen Gesellschaft. Zu Riam-Kanan in Süd-Kalimantan, wo ich aufenthalte, hörte ich zufälligerweise ein Gerücht, das ein geschwänztes Kind lebte. Herr K. aus Bali sprach fliessend. Englich und suchte das Kind auf meinen eigenen Wunsch auf. Am 25. 7. 1971 fuhr ich mit ihm von dort $200 \mathrm{~km}$ entfernt bei Amntei wie eine Expedition. In entlegener Landgegend (Dorf B.) kannten wir einen Onkel des Kindes. In dieser Gegend sind nach Herr K. fanatische mahommedaner. Durch seine Führung besuchten wir durch einen Kahn das Kind. Der $3 \frac{1}{3}$ Jahre alte Knabe wohnte mit seiner Familie zu einem 2 Wohnzimmerhaus entlang eines Nebenflusses Barito. Der Kleine Junge (Name:N.) war das erste Kind einer 35 jährigen Frau. Sein Vater ist 45 Jahre alt. Über mögliche Konsanguinitait oder erbliche Krankheiten war nichts bekannt. Meine Frage, ob seine Vorfahren Dajak waren, verneinte seine Mutter. Die Schwangerschaft verlief normal und die Entbindung war glatt. Die körperliche und geistige Entwicklung des Kindes war normal. Die Körperlänge betrug $98 \mathrm{~cm}$. Es zeigte keine Missbildung, insbesondere liess sich an der Wirbelsäule irgend eine krankhafte Veränderung nicht nachweisen.

Der Schwanz war $12 \mathrm{~cm}$ lang und hatte an der dicksten Stelle einen Durchmesser von $2 \mathrm{~cm}$. An der Basis des Schwanzes zeigte sich eine Einschnürung im Durchmesser von $1 \mathrm{~cm}$. Das Gebilde fühlte sich vollständig weich und schlaff an. Es liess keinerlei muskuläre oder gar knöcherne Anteil tasten. Seine Abgangsstelle befand sich oberhalb 


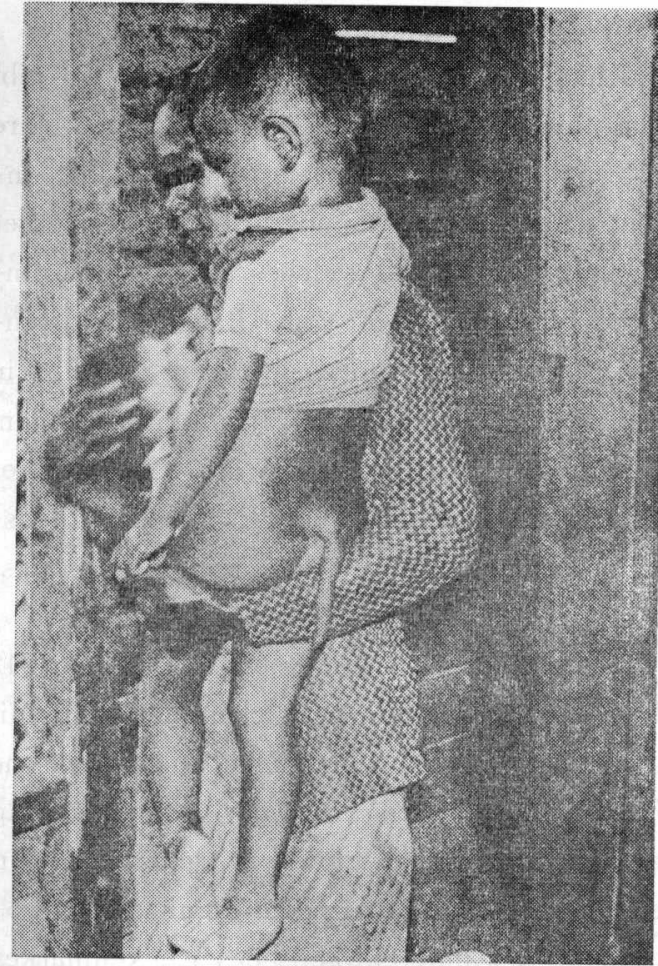

Abbildung 1 Geschwänzter Knabe und seine Mutter

der Gesässspalte etwas rechtswärts an dem Fovea coccygea. (Abb. 1 u. 2) Mein Vorschlag der operativen Entfernung des Schwanzes wurde von der Familie verweigert, weil der Schwanz die Gottesgabe war. Das Kind war ein Wunderkind und wird in Zukunft ein Wahrsager. Dabei spielt gewiss religiöser Grund eine Rolle.

\section{Diskussion und Literaturübersicht}

Wenn ich diese eben beschriebene Schwanzbildung unter die von Bartels angegebenen Fälle unterbringen möchte, so gehört dieselbe unter die vierte Form. Es handelt sich hier um einen sogenannten Schweineschwanz, der sich durch den Mangel irgendwelches differnzierbaren Gewebes auszeichnet, wie Schwarz (1912) usw. eine ähnliche Beobachtung berichten.

Bartels ist der erste Mann, im Jahre 1884 aus dem Schriftum 116 Fälle zusammenzuste-

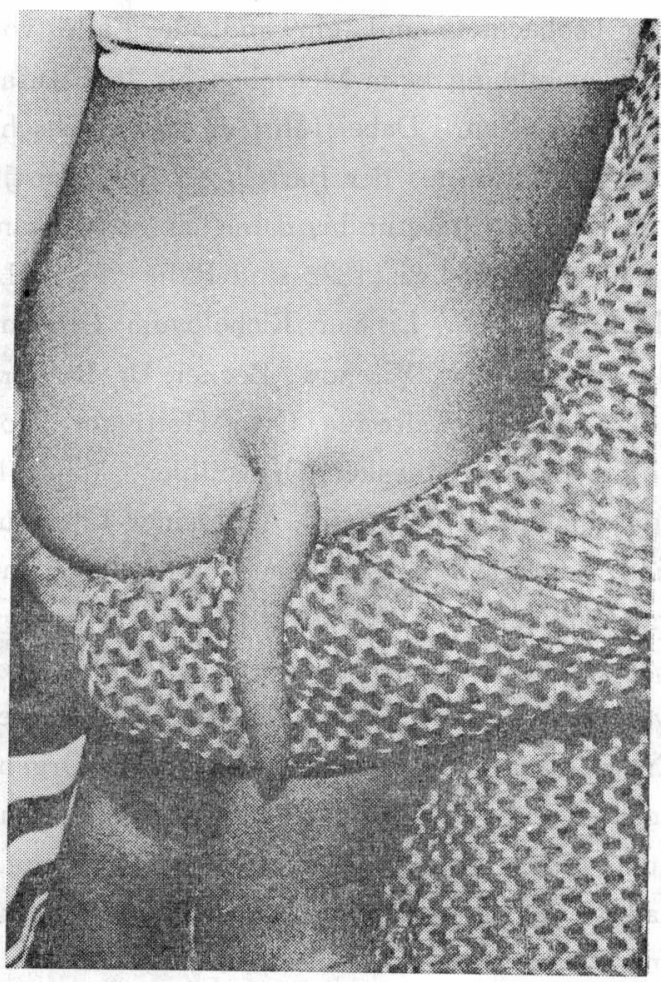

Abbildung 2 Vergrösserungsaufnahme des Schwanzes beim Kind

llen. In 48 von diesen Fällen war das Geschlecht nicht angegeben, in 52 Fällen war es männlich und in 16 weiblich. Die Literatur über diesen Gegenden nach dem Jahre 1884 ist indes eine sehr geringe.

Im Jahre 1884 findet sich ein geschwänztes: Kind im Bericht von Virchow. Dieses Kind hatte eine Schwanzbildung, die unter der $\mathrm{Ka}$ tegorie "weiche Schwänze" (Bartels' Einteilung Nr. IV). Virchow hat 2 grosse Gruppen zu scheiden vorgeschlagen, je nachdem der betreffende Caudalanhang Wirbel oder Wirbelrudimente enthielt, oder nicht; er schlug vor die zur ersteren Gruppe gehörenden als "Wirbelschwänze" (komplete Schwänze), die übrigen als "weiche Schwänze"(imkomplete Schwänze) zu bezeichnen.

Dann berichtet Schaeffer, im ganzen 93 si- 
cher beobachtete und einwandfreie Fälle von Schwanzbildung beim Menschen bis 1892 zusammenzustellen. Dabei führt er die bestbeschriebenen Fälle aus der Bartels' schen Casuistik fort, um die Literatur bis dahin zu vervollständigen. Seit 1884 bis 1892 sind Fälle (Freund, Henning-Rauber, Lissner, Eichelbaum, Obolonsky, Sonnenburg, Virchow, Becker, De Ruyter, Fol, Snowball, Laforgue, Otto, Hottinger, Hohlfeld, Sachse, Huesker, Cruveilhier) ihm als neu zugänglich gewesen. Aus seiner Literatur sind Schwanzbildungen beim Menschen sind meist mit anderen Bildungs-Anomalien gepaart. $1 / 3$ der Fälle lassen sich weitere mehr oder weniger schwere Missbildungen, besonderes Entwicklungsstörungen des anorektalen Organs nachweisen. Die Ursache der Amnionverbindung (z. B. Adhäsion) erzeugt eine Störung, das ist die Schwanzbildung, meint er im frauenärztlichen Fachkenntnis.

Im Jahre 1896 berichtet Waldeyer über einen $4 \mathrm{~cm}$ langen, weichen Schwanz eines 5 Wochen alten Knaben. Er untersuchte zum Vergleiche nun den Bau eines Schweineschwanzes und eineṣ Lämmerschwanzes, in welchem sich keinerlei Hartgebilde, weder Knorpel noch Knochen, mehr finden. Bei den beiden Vergleichsobjekten in Querschnitte zeigten letztere ganz den gleichen Bau wie seiner beschriebenen menschlichen Caudalanhang; insbesondere zeigt der Schweineschwanz eine grosse Ähnlichkeit mit derartigen weichen menschlichen Caudalanhängen in feineren Strukturverhältnissen sehr wohl.

Am Anfang der 20. Jahrhundertes berichtet Harrison (1902) über einen $5,3 \mathrm{~cm}$ langen weichen Schwanz eines 3 Monatigen alten Knaben. Das merkwürdige Merkmal des Schwanzes ist seine Motilität. Nach der operativen Entfernung des Schwanzes fanden sich einige gestreifte Muskulaturen in Querschnitten. Knochengewebe ist nicht vorhanden. Es gibt auch eine russische Literatur aus dem Jahre 1893 von Piatnitzky, der in einem Kaudalanhang eines jungen Mannes “Bündel gestreifter Muskelfasern antraf, welches auf das ursprüngliche Vorhandensein von Ursegmenten hin-. deutet". Harrison untersucht den Schwanz in den Menschenembryonen. In $14 \mathrm{~mm}$ langem Menschenembryo stellt er das Gebilde des Schwanzes regelmässig dar. Es persistiert anstatt zu schrumpfan und zu schwinden, so dass dasselbe zu einem Schwanz wird.

Konstantinowicz beschreibt im Jahre 1912 über ein totgeborenes weibliches Monstrum mit einem weichen Schwanz. Die mikroskopische Untersuchung erwiess, dass der zentrale Strang des Schwanzes nicht blos mit dem Steissbein verwachsen war, sondern die unmittelbare Fortsetzung des unteren Teils des Rückenmarkes darstellte : infolgedessen rechnet er diesen Schwanz zu den echten menschlichen Schwänzen, obzwar derselbe der Wirbel entbehrte.

Schwarz berichtet im Jahre 1912 einen Fall der Gruppe IV. Es war ein gut entwickelter, männlicher Neugeborener mit einem $51 / 2 \mathrm{~cm}$ langen, bis $\mathrm{zu} 1 \mathrm{~cm}$ dicken Schwanz ohne sonstige Missbildungen. Nach der Entfernung zeigte sich bei der mikroskopischen Untersuchung ausser einer starken zentralen Arterie keinen besonderen Befund. Das Kind starb 2 Monate später an einer Knieeiterung. Die $\mathrm{Au}-$ topsie zeigte sonst vollkommen normale Verhältnisse. Durch seinem Schlusswort scheint das männliche Geschlecht den Berichten nach von der Schwanz-bildung bevorzugt zu sein. Auch hier ist die Schwanzbildung bei einem. männlichen Individuum beobachtet worden.

Rostock (1927) veröffentlicht Beobachtung eines geschwänzten weiblichen Neugeborenen 
Kindes in Schweineschwanzform. Das Schwänzchen zeigte eine gekrümmte Spitze. Die Basis des Schwänzchens im Durchmesser von $1.2 \mathrm{~cm}$ befand sich in Höhe der Spina iliacae anterior superior und hatte nicht die geringste Verbindung zur Wirbelsäule. Bei der Betrachtung der Schnitte bestand aus Haut mit Haaren und Drüsen ohne Besonderheiten. Im Innern befanden sich Fettgewebe, durchgezogen von Bindegewebssepten sowie Nerven und Gefässe : Knochengewebe oder Knorpelgewebe waren nicht nachzuweisen. Rostock bezeichnet die Ätiologie der von ihm beschriebenen Schwanzbildung als ungeklärt.

Jorns publiziert im Jahre 1955 eine ähnliche Mitteilung. Es war ein gesundes und von weiteren Fehlbildungen freies Mädchen im Alter von 3 Monaten mit einem $51 / 2 \mathrm{~cm}$ langen, $1 \mathrm{~cm}$ dicken Schwanz. Seine Abgangsstelle befand sich in Höhe des Dornfortsatzes von L.W.S.V. Die Röntgenuntersuchung der Wirbelsäule ergab keine abweichenden Veränderungen. Mikroskopisch fand sich in Übersichtsschnitten regelrechte Haut, darunter lockeres Bindegewebe mit atrophischen Hautanhangsgebilden, sonst lediglich Fettgewebe, das von einem grösseren Gefässbündel durchzogen wird. Knochengewebe ist nicht vorhanden.

In letzter Zeit berichtet Martinho (1955) in Brasilien über ein weibliches neugeborenen Kindes mit einem $12 \mathrm{~cm}$ langen weichen Schwanz ohne muskulären und knöchernen Inhalt. Der Schwanz wurde vom Autor erfolgreich operiert.

Dann berichtet Battaglia (1958) über einen $4.5 \mathrm{~cm}$ langen, weichen Schwanz eines Knaben im Alter von 3 Jahre ohne knöchernen Inhalt. Der Knabe hatte Mikromelia sinistra. Battaglia schildert ' $L$ ' Ac sarebbe da interpretare come un arresto di sviluppo, a carattere talora eredi- tario".

Im Jahre 1960 berichtet Rijsbosch, über einen operierten Fall von einer Menschenschwanzformation bei einem neugeborenen Mädchen. Das Schwänzchen sass an der coccygealen Region.

Für die Entstehungsweise der weichen Schwanze ist, soweit ich sehe, bisher keine einleuchtende "Erklärung" gegeben worden. Schierhorn (1968) berichtet über zwei Beobachtungen persistierender Rumpfschwanzrnospen bei neugeborenen Knaben. In beiden Fällen ist die Persistenz der embryonalen Rumpfschwanzknospe möglicherweise bedingt durch eine frühzeitige Verwachsung mit dem Damm.

\section{Geographische Verbreitung der geschwä- nzten Menschen}

Ein "geschwanztes Volk" ist bis jetzt nicht gefunden, obwohl der geschwänzte Mensch in dem Bericht von Bartels über die ganze Erde verbreitet ist.

Bartels beschreibt über Menschen mit Schwanzbildung auf Borneo (jetzt grosser Teil Kalimantan) 12 Seiten lang in seinem Referat. Damals fanden sich mehrere holländische $\mathrm{Be}-$ obachtungen mit Schwanzen auf Borneo. Die Beobachtungen, welche uns durch Reisende überbracht sind, sind so äusserlich und wenig planmässig, so von vorgefassten Ansichten durchtränkt, gemacht: worden, dass sie sehr schwer zu einem pro oder contra verwertet werden können. Deshalb gab es das negative Urteil der Beschreibung.

Ich habe selber den Eindruck in meinem Aufenthalt in Süd-Kalimantan (Borneo), dass mindestens der Glaube an die Exsistenz geschwänzter Menschen auf Borneo (Kalimantan) bei der Bevölkerung dieser Insel ein allgemein verbreiteter und festwurzelnder ist. 


\section{Zusammenfassung}

Es wird ein $31 / 3$ jähriges männliches Kind mit einem $12 \mathrm{~cm}$ langen, $2 \mathrm{~cm}$ dicken Schwanz in Süd-Kalimantan im Licht der einschlägigen Literaturen beschrieben. [Ich habe einen Vortrag über diesen Fall am 1.11. 1971 in der 36. Sitzung (Human Ecology and Race Hygiene) gehalten]

\section{Schriftum}

Bartels, M. : Die geschwänzten Menschen, A.f. Anthropol. 15, 45-131 (1884)

Virchow, R.: Schwanzbildung beim Menschen, Berl. Klin. Wschr. 21, 745-747 (1884)

Schaeffer, O.: Beitrag zur Ätiologie der Schwanzbildung beim Menschen, A.f. Anthropol. 20, 189 -224 (1892)

Freund, H. W.: Über Schwanzbildung beim Menschen, Virch. Arch., 104, 531-539 (1886)

Henning, C. u. Rauber, A. : Ein neuer Fall von geschwänzten Menschen, Virch. Arch., 105, 83109 (1886)

Waldeyer, W.: Die Caudalanhänge des Menschen, Sitzungsber. K. preuss. Acad. Wiss., 2, 775-784 (1896)

Harrison, R. G.: Occurence of tail in man, with a description of the case reported by Dr. Watson, Johns Hopkins Hosp. Bull., 12, 96-101 (1901)

Piatnitzky, J. J.: Zur Frage über Schwanzbildung beim Menschen, Inaug. Diss. Moskau 1893 (zit. Waldeyer)

Konstantinowicz, V.: Zur Frage der Schwanzbildung beim Menschen, Z.f. Heilk., 28, 1-28(1907)

Schwarz, G.: Beitrag zur Kenntnis des geschwänzten Menschen, M. M. W., 59, 928-929 (1912)

Rostock, P.: Beitrag zur Kasuistik des geschwänzten Menschen, Beitr. klin. Chir., 138, 657-662 (1927)

Jorns, G.: Über die Schwanzbildung beim Menschen, Zbl. Allg. Path. Anat., 93, 259-261 (1955)

Martinho Da Rocha J. Orlandi, O. V.: Persistēncia de cauda embrionária em recémnascido (Persistence of the embryonic tail in newborns) Bol. Inst. Puericult. Univ. Brasil, 12/2, 81-95 (1955)

Battaglia, S. Parisio, B.: Sulla Appendice caudale Nell' Uomo (A caudal Appendix in man), Folia hered. Path. (Pavia), 71, 1-6 (1958)

Rijsbosch, J. K.: Tail formation in man, Arch. Chir. Neerl. 12/2, 216-219 (1960)

Schierhorn, H.: Über die Textur der persistierenden Rumpfschwanzknospe beim Menschen, $Z$. Mikrosk. Anat. Forsch. 79, 244-257 (1968) 